\title{
Electrical characteristics of high-energy proton irradiated ultra-thin gate oxides
}

\author{
J.M. Rafí, B. Vergnet, F. Campabadal, C. Fleta, L. Fonseca, \\ M. Lozano, C. Martínez, M. Ullán \\ Institut de Microelectrònica de Barcelona (IMB), CNM-CSIC, Campus UAB, 08193 Bellaterra, Spain.
}

\begin{abstract}
This paper presents the first results of an investigation of the effect of high-energy proton irradiations on the electrical characteristics of ultra-thin gate oxides. By means of the analysis of the electrical conduction in irradiated MOS capacitors, it is shown that the radiation-induced leakage current is similar to the one obtained by electrical stress. In addition, the damage created in the oxide by proton radiation has a similar nature, as revealed by electrical stressing of irradiated devices.
\end{abstract}

\section{Introduction}

The study of ionizing radiation effects on thin gate oxides has recently received increased attention due to the introduction of new techniques such as X-ray and e-beam lithography in ULSI technologies that use ultra-thin gate dielectrics. In addition, for applications in space or in high-energy physics such as in the future Large Hadron Collider at CERN in Geneva, CMOS devices of read-out circuitry will be operating in a very hostile radiation environment.

The results of recent investigations have shown that ionizing radiation damages the oxide leading to a radiation-induced leakage current (RILC). It has been attributed to the same physical mechanism as when electrically stressing the MOS devices, that gives rise to the well-known Stress Induced Leakage Current (SILC) [1-4]. As a consequence, it represents a limiting factor to MOS device reliability.

Results have been reported on effects on thin oxides subjected to radiation from different sources as gamma rays, X-rays, electrons or heavy ions. But, to our knowledge, the effect of high-energy proton irradiation on ultra-thin oxides has not been studied. In this work the radiation-induced effects on the electrical properties of ultra-thin gate oxides irradiated by highenergy protons are investigated. The degradation observed is compared with the one resulting from electrical stress through ramped gate voltage sequences.

\section{Test structures and experimental procedure}

The devices used in this work were manufactured at IMB-CNM. They consist of square shaped MOS capacitors with an area of $\mathrm{A}=6.4 \times 10^{-5} \mathrm{~cm}^{2}$. The starting material was $<100>$ p-type $\mathrm{CZ}$ silicon wafers with a boron concentration of about $10^{15} \mathrm{at} / \mathrm{cm}^{3}$. After definition of the active regions in a $400 \mathrm{~nm}$-field oxide, the gate oxide was grown in a $\mathrm{N}_{2} / 10 \% \mathrm{O}_{2}$ ambient at $800^{\circ} \mathrm{C}$, leading to a final $\mathrm{SiO}_{2}$ thickness $\left(\mathrm{t}_{\mathrm{ox}}\right)$ of $4.9 \mathrm{~nm}$. A highly doped n-type polysilicon layer with a thickness of $350 \mathrm{~nm}$ was used for the gate material and covered also the transition between gate and field oxides. 


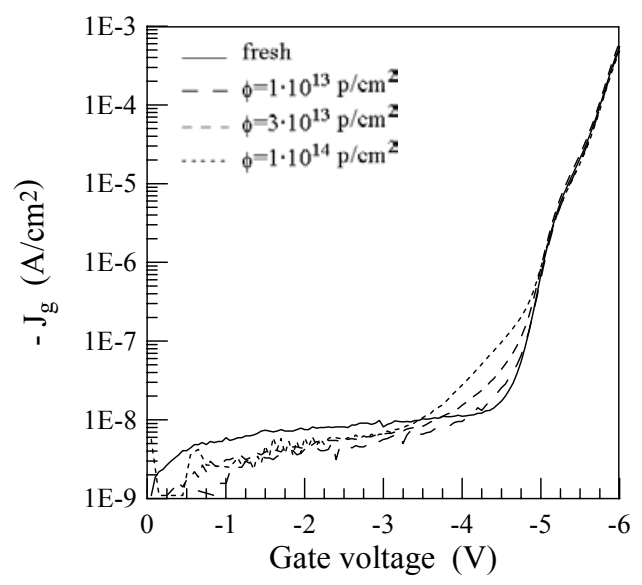

Fig 1. $\mathrm{J}_{\mathrm{g}}$ vs. $\mathrm{V}_{\mathrm{g}}$ curves for negative polarization, for fresh and proton-irradiated MOS capacitors.

An aluminium pad in the field region allowed for contacting to the polysilicon gate layer.

Devices were irradiated with high-energy (24 $\mathrm{GeV}$ ) protons, at the CERN Proton Synchroton beam. Pieces of the wafers containing the MOS capacitors under investigation were exposed to three different proton irradiation fluences: $\phi=10^{13}, 3 \times 10^{13}$ and $10^{14}$ protons $/ \mathrm{cm}^{2}$, which were achieved in single irradiation steps. The proton fluence was measured by activation analysis of an aluminium foil, with the same size of the samples, placed close to them, in the same position with respect to the proton beam, so as to measure the same average fluence received by the MOS capacitors. Since the devices were not packaged, no bias was applied during irradiation. After irradiation and before any electrical measurement was carried out, the samples were kept at a temperature of $-18^{\circ} \mathrm{C}$.

To investigate the impact of the irradiation on the gate oxide, the gate current versus gate voltage $\left(\mathrm{I}_{\mathrm{g}}-\mathrm{V}_{\mathrm{g}}\right)$ characteristics have been measured for positive and negative polarization, i.e., gate voltage positive or negative with respect to the silicon substrate. Ramped $\mathrm{I}-\mathrm{V}$ measurements were performed at room temperature, in a light-proof and electrically shielded probe station using an HP 4155B parameter analyzer.

\section{Results}

\subsection{J-V characteristics of irradiated devices}

The measured $J_{g}-V_{g}\left(J_{g}=I_{g} / A\right)$ characteristics for non-irradiated and irradiated MOS capacitors are shown in Fig. 1 and 2, for negative and positive gate

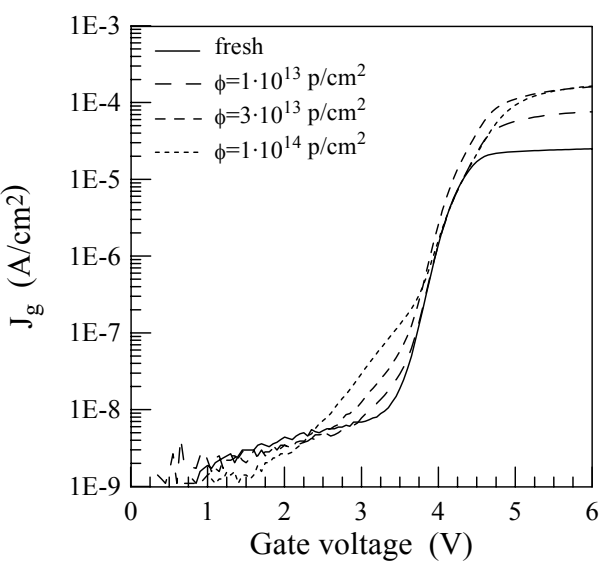

Fig 2. $J_{\mathrm{g}}$ vs. $\mathrm{V}_{\mathrm{g}}$ curves for positive polarization, for fresh and proton-irradiated MOS capacitors.

voltages, respectively. For the non-irradiated samples, the expected J-V characteristics are obtained. In the case of negative bias, two regions are clearly distinguished. The first one corresponds to low gate voltages $\left(\left|\mathrm{V}_{\mathrm{g}}\right|<4.5 \mathrm{~V}\right)$ and is characterised by a low gate current $\left(\left|I_{g}\right|<1 p A\right)$. As $V_{g}$ is increased, the device enters a second region in which the gate current density is exponentially dependent on the oxide voltage and corresponds to Fowler-Nordheim tunnelling conduction through the gate oxide. For positive bias, the tunnelling conduction starts at voltages close to $3.2 \mathrm{~V}$, and the current increases until a saturation occurs due to the lack of free electrons at the silicon substrate in this biasing condition.

For the proton-irradiated samples, the conduction characteristics are similar to those of fresh devices, except for the low-oxide field region in which a clear increase in gate current is observed, as shown in Figs. 1 and 2. This increase in leakage current with the proton fluence corresponds to the Radiation Induced Leakage Current (RILC) phenomenon. In order to better analyse the effect of the proton irradiation on the leakage current, the fractional excess current $\left(\Delta \mathrm{J}_{\mathrm{g}} / \mathrm{J}_{\mathrm{g} 0}=\left(\mathrm{J}_{\mathrm{g}}-\mathrm{J}_{\mathrm{g} 0}\right) / \mathrm{J}_{\mathrm{g} 0}\right.$, with $\mathrm{J}_{\mathrm{g} 0}$ the current for the fresh device) is plotted in Fig. 3. The curves peak at the same voltage regardless of the proton fluence for each biasing condition, with a higher peak as the irradiation fluence increases. For negative voltages, the curves peak at $\sim-4.5 \mathrm{~V}$, whereas for positive bias the peak is at $\sim 3.5 \mathrm{~V}$. These values correspond to the same oxide voltage, thus indicating that the traps created by the irradiation are responsible for the excess leakage current. 


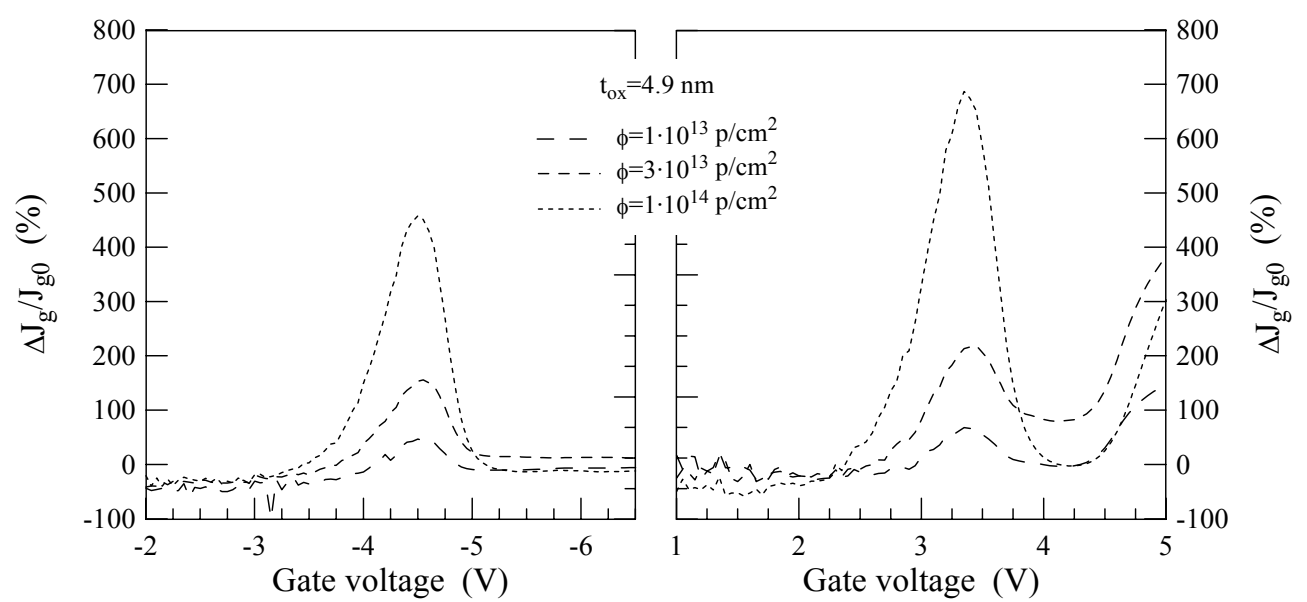

Fig. 3. Fractional excess gate current vs. gate voltage for proton-irradiated MOS devices with an oxide thickness of $4.9 \mathrm{~nm}$. For negative bias curves peak at $\mathrm{V}_{\mathrm{g}} \sim-4.5 \mathrm{~V}$, and for positive bias the peak is at $\mathrm{V}_{\mathrm{g}} \sim 3.5 \mathrm{~V}$, for the three fluences analysed.

\subsection{Electrical stress induced leakage current}

In order to analyse if there is a correlation between RILC and SILC in our proton-irradiated devices, non-irradiated samples were electrically stressed by means of consecutive negative voltage sweeps from $0 \mathrm{~V}$ to up to different and increasing gate voltages.

In Fig. 4 the obtained results are shown. We also observe in this case a SILC component in the total current, which increases as the maximum voltage increases. In our case, however, no noticeable instabilities exist as reported in [1]. After a first voltage sweep stressing, consecutive I-V measurements up to the same voltage do not show further current differences. This is clearly observed in Fig. 5 where the fractional excess leakage current is represented as function of the gate voltage. Consecutive I-V measurements up to $-6 \mathrm{~V}$ lead to the same SILC values and it is only when the maximum voltage is increased that the SILC also increases. This could be interpreted as if saturation in the degradation of the oxide is achieved depending on the electrical stress conditions, so that only when increasing the stressing magnitude a further degradation occurs. In the same figure, the RILC results are also shown. The close correlation between the SILC and RILC not only quantitatively, but also the dependence on the gate voltage indicates that the same type of physical damage is created in the thin oxide whether electrically stressing or by high-energy proton radiation. As an example, a proton fluence of $10^{14}$ $\mathrm{p} / \mathrm{cm}^{2}$ leads to the same electrical damage as a voltage sweep up to $-6.5 \mathrm{~V}$.

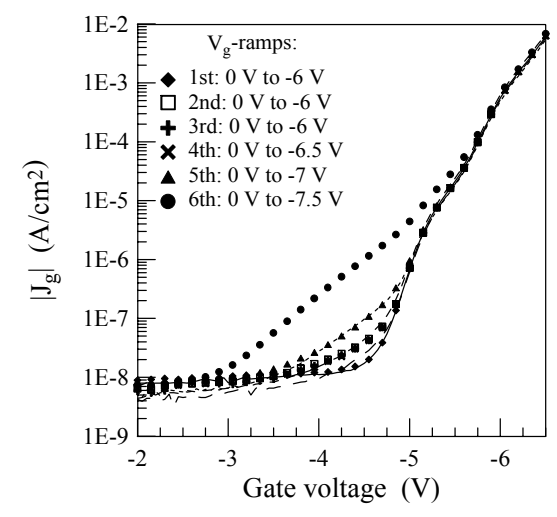

Fig. 4. $\mathrm{J}_{\mathrm{g}}$ vs. $\mathrm{V}_{\mathrm{g}}$ curves for electrically stressed capacitors through consecutive voltage ramps (symbols) and for irradiated devices (lines)

A further confirmation of the similarities between SILC and RILC for high-energy protons is given by the results obtained when doing SILC experiences on irradiated devices. In Fig. 6 results of consecutive I-V measurements corresponding to samples irradiated with a proton fluence of $10^{14} \mathrm{p} / \mathrm{cm}^{2}$ are given. These results show that when electrically stressing up to -6.5 $\mathrm{V}$ no further increase in the leakage current is obtained. 


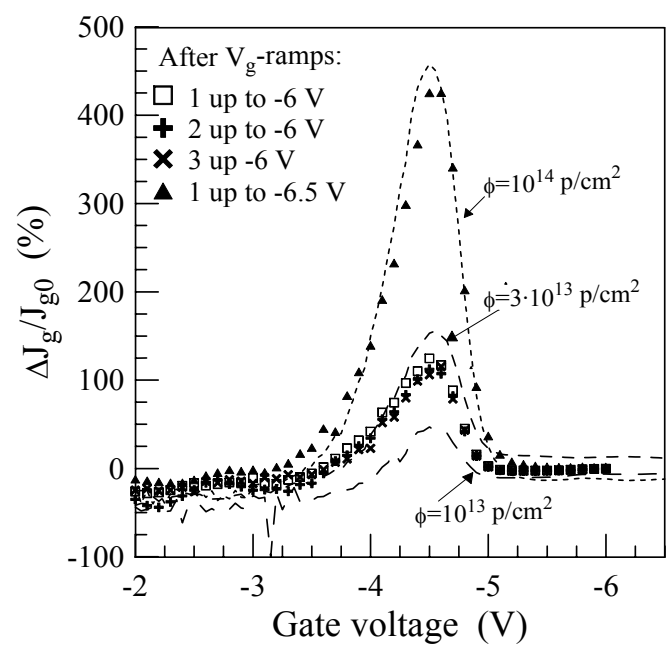

Fig. 5. Fractional excess current for electrically stressed and irradiated samples.

It indicates that this stressing also represents a saturation in terms of electrical degradation. It is only when an electrical stress of higher magnitude (voltage sweep up to $-7 \mathrm{~V}$ ) is performed that a SILC component appears.

\section{Conclusion}

Ultra-thin gate oxides irradiated by high-energy protons show an increased leakage current in the lowfield region, for both biasing polarities. This effect is similar to those reported for other radiation sources, giving rise to the so-called radiation-induced leakage current. The fractional excess current exhibits a peak for the same oxide voltage in both polarities. This suggests that the damage caused by proton irradiation can be modelled by a trap level in the oxide, which leads to a trap-assisted tunnelling conduction mechanism dominating at low fields.

In addition, comparison with the results obtained when electrically stressing the oxide with voltage ramps, further confirm the similar nature of the damage created by either irradiation or electrical stress. A more complete analysis is needed, however, to gain a deeper insight of the degradation mechanism and the created damage.

\section{Acknowledgements}

This work has been partially supported by the Comisión Interministerial de Ciencia y Tecnología

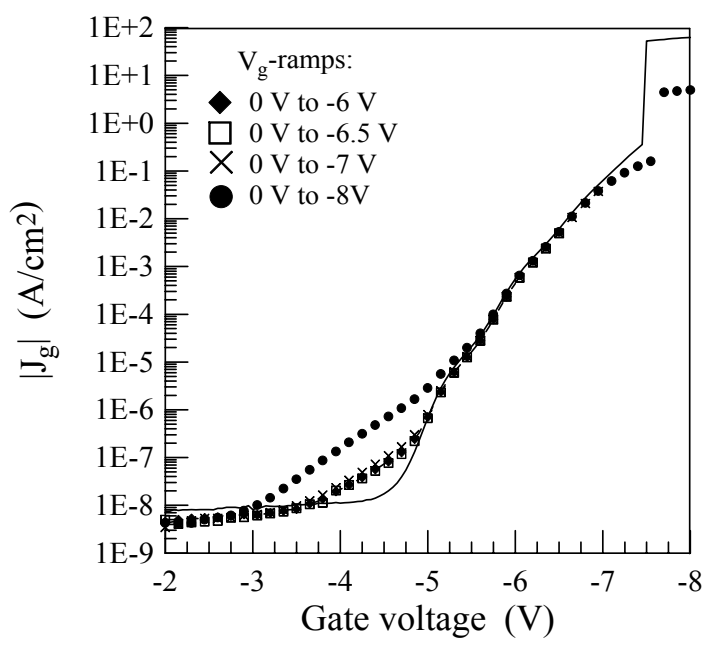

Fig. 6. $J_{g}$ vs. $V_{g}$ curves for electrically stressed capacitors previously irradiated with a proton fluence of $10^{14} \mathrm{p} / \mathrm{cm}^{2}$.

(CICYT) under project FPA2000-1560-C02-02.

\section{References}

[1] Scarpa A, Paccagnella A, Montera F, Ghibaudo G, Pananakakis G, Ghidini G and Fuochi PG. Ionizing radiation induced leakage current on ultra-thin gate oxides. IEEE Trans. Nucl. Sci. 44 (1997) 1818-1825.

[2] Ceschia M, Paccagnella A, Sandrin S, Ghidini G, Wyss J, Lavale $\mathrm{M}$ and Flament O. Low field leakage current and soft breakdown in ultra-thin gate oxides after heavy ions, electrons or X-ray irradiation. IEEE Trans. Nucl. Sci. 46 (2000) 566-573.

[3] Kim SJ, Cho BJ, Chong PF, Chor EF, Ang CH, Ling $\mathrm{CH}$, Joo MS and Yeo IS. Does short wavelength lithography degrade the integrity of thin gate oxide? Microelectronics Reliability 40 (2000) 1609-1613.

[4] Cho BJ, Kim SJ, Ling CH, Joo MS and Yeo IS. A comparison between leakage currents in thin gate oxides subjected to X-ray radiation and electrical stress degradation. Solid-State Electron. 44 (2000) 12891292. 